Honam Mathematical J. 34 (2012), No. 1, pp. 045-054

http://dx.doi.org/10.5831/HMJ.2012.34.1.45

\title{
STABILITY OF MULTIPLICATIVE INVERSE FUNCTIONAL EQUATIONS IN THREE VARIABLES
}

\author{
Eun Hwi LeE
}

\begin{abstract}
In this paper, we prove stabilities of multiplicative functional equations in three variables such as

$$
\begin{aligned}
& r\left(\frac{x+y+z}{3}\right)-r(x+y+z) \\
& =\frac{2 r\left(\frac{x+y}{2}\right) r\left(\frac{y+z}{2}\right) r\left(\frac{z+x}{2}\right)}{r\left(\frac{x+y}{2}\right) r\left(\frac{y+z}{2}\right)+r\left(\frac{y+z}{2}\right) r\left(\frac{z+x}{2}\right)+r\left(\frac{z+x}{2}\right) r\left(\frac{x+y}{2}\right)}
\end{aligned}
$$

and

$$
\begin{aligned}
& r\left(\frac{x+y+z}{3}\right)+r(x+y+z) \\
& =\frac{4 r\left(\frac{x+y}{2}\right) r\left(\frac{y+z}{2}\right) r\left(\frac{z+x}{2}\right)}{r\left(\frac{x+y}{2}\right) r\left(\frac{y+z}{2}\right)+r\left(\frac{y+z}{2}\right) r\left(\frac{z+x}{2}\right)+r\left(\frac{z+x}{2}\right) r\left(\frac{x+y}{2}\right)} .
\end{aligned}
$$

In 1940, Ulam [16] proposed the Ulam stability problem of additive

Let $G_{1}$ be a group and let $G_{2}$ be a metric group with a metric $d(\cdot, \cdot)$. Given $\epsilon>0$, does there exist a $\delta>0$ such that if a mapping $h: G_{1} \rightarrow G_{2}$ satisfies the inequality $d(h(x y), h(x) h(y))<\delta$ for all $x, y \in G_{1}$, then there exists a homomorphism $H: G_{1} \rightarrow G_{2}$ with $d(h(x), H(x))<\epsilon$ for all $x \in G_{1}$ ?
\end{abstract}

\section{Introduction} mappings;

In 1941, Hyers [6] answered the Ulam's question for the case of the additive mapping on the Banach spaces.

Let $G_{1}$ and $G_{2}$ are Banach spaces. Assume that a mapping $f: G_{1} \rightarrow G_{2}$ satisfies the inequality

$$
\|f(x+y)-f(x)-f(y)\| \leq \varepsilon
$$

Received November 15, 2011. Accepted December 7, 2011. 2000 Mathematics Subject Classification. 39B72, 39B22.

Key words and phrases. Functional Equation, Stability. 
for all $x, y \in G_{1}$. Then the limit $g(x):=\lim _{n \rightarrow \infty} \frac{f\left(2^{n} x\right)}{2^{n}}$ exists for all $x \in G_{1}$ and $g$ is the unique additive mapping satisfying

$$
\|f(x)-g(x)\| \leq \varepsilon
$$

for all $x \in G_{1}$.

In 1978, Th. M. Rassias [13] generalized the above result of an approximation involving a sum of norms;

Let $G_{1}$ be a vector space and $G_{2}$ a Banach space. Assume that a mapping $f: G_{1} \rightarrow G_{2}$ satisfies

$$
\|f(x+y)-f(x)-f(y)\| \leq \varepsilon\left(\|x\|^{p}+\|y\|^{p}\right)
$$

for all $x, y \in G_{1}, \varepsilon>0$ and $p<1$. Then the limit $g(x):=$ $\lim _{n \rightarrow \infty} \frac{f\left(2^{n} x\right)}{2^{n}}$ exists for all $x \in G_{1}$ and $g$ is the unique additive mapping satisfying

$$
\|f(x)-g(x)\| \leq \frac{2 \varepsilon}{2-2^{p}}\|x\|^{p}
$$

for all $x \in G_{1}$.

During the last two decades a number of papers and research monographs have been published on various generalizations and applications of the generalized Hyers-Ulam stability to a number of functional equations and mappings (see [1]-[16]). P. Găvruta [4] provided a further generalization of Th. M. Rassias' Theorem.

In this paper, we investigate stabilities of another type functional equations in three variables such as

$$
\begin{aligned}
& r\left(\frac{x+y+z}{3}\right)-r(x+y+z) \\
& =\frac{2 r\left(\frac{x+y}{2}\right) r\left(\frac{y+z}{2}\right) r\left(\frac{z+x}{2}\right)}{r\left(\frac{x+y}{2}\right) r\left(\frac{y+z}{2}\right)+r\left(\frac{y+z}{2}\right) r\left(\frac{z+x}{2}\right)+r\left(\frac{z+x}{2}\right) r\left(\frac{x+y}{2}\right)}
\end{aligned}
$$

and

$$
\begin{aligned}
& r\left(\frac{x+y+z}{3}\right)+r(x+y+z) \\
& =\frac{4 r\left(\frac{x+y}{2}\right) r\left(\frac{y+z}{2}\right) r\left(\frac{z+x}{2}\right)}{r\left(\frac{x+y}{2}\right) r\left(\frac{y+z}{2}\right)+r\left(\frac{y+z}{2}\right) r\left(\frac{z+x}{2}\right)+r\left(\frac{z+x}{2}\right) r\left(\frac{x+y}{2}\right)} .
\end{aligned}
$$

Since $\frac{1}{x}$ is a solution of these equations, these called multiplicative inverse functional equations in three variables. Also the solution of equation (1.1) and (1.2) are called multiplicative inverse mappings. Note 
that reciprocal difference functional equations

$$
r\left(\frac{x+y+z}{3}\right)-r(x+y+z)=\frac{2 r(x) r(y) r(z)}{r(x) r(y)+r(y) r(z)+r(z) r(x)}
$$

and reciprocal adjoint functional equations in three variables

$$
r\left(\frac{x+y+z}{3}\right)+r(x+y+z)=\frac{4 r(x) r(y) r(z)}{r(x) r(y)+r(y) r(z)+r(z) r(x)} .
$$

are similar types with multiplicative functional equations in three variables. Stabilities of reciprocal difference functional equation and the reciprocal adjoint functional equation in two variables was proved by $\mathrm{K}$. Ravi, J. M. Rassias and B. V. Senthil Kumar [15]. And Y. W. Lee and G. H. Kim [14] extend these equations to m-variables. But we investigate stabilities of another type functional equations in three variables.

\section{Stabilities}

Define for all $x, y, z$ in $R$

$$
\begin{aligned}
D_{r}(x, y, z)= & r\left(\frac{x+y+z}{3}\right)-r(x+y+z) \\
& -\frac{2 r\left(\frac{x+y}{2}\right) r\left(\frac{y+z}{2}\right) r\left(\frac{z+x}{2}\right)}{r\left(\frac{x+y}{2}\right) r\left(\frac{y+z}{2}\right)+r\left(\frac{y+z}{2}\right) r\left(\frac{z+x}{2}\right)+r\left(\frac{z+x}{2}\right) r\left(\frac{x+y}{2}\right)}
\end{aligned}
$$

and

$$
\begin{aligned}
E_{r}(x, y, z)= & r\left(\frac{x+y+z}{3}\right)+r(x+y+z) \\
& -\frac{4 r\left(\frac{x+y}{2}\right) r\left(\frac{y+z}{2}\right) r\left(\frac{z+x}{2}\right)}{r\left(\frac{x+y}{2}\right) r\left(\frac{y+z}{2}\right)+r\left(\frac{y+z}{2}\right) r\left(\frac{z+x}{2}\right)+r\left(\frac{z+x}{2}\right) r\left(\frac{x+y}{2}\right)} .
\end{aligned}
$$

Theorem 1. Let $X$ and $Y$ be spaces of non-zero real numbers. Assume that $f: X \rightarrow Y$ satisfies the functional inequality

$$
\left|D_{f}(x, y, z)\right| \leq \phi(x, y, z)
$$

for all $x, y, z \in X$, where $\phi: X^{3} \rightarrow Y$ is a function such that for all $x, y, z \in X$

$$
\Phi(x, y, z):=\sum_{i=0}^{\infty} \frac{1}{3^{i}} \phi\left(\frac{x}{3^{i+1}}, \frac{y}{3^{i+1}}, \frac{z}{3^{i+1}}\right)<\infty .
$$


Then there exists a unique multiplicative inverse mapping $A: X \rightarrow Y$ which satisfies (1.1) and

$$
|f(x)-A(x)| \leq \Phi(x, x, x)
$$

for all $x \in X$.

Proof. Replacing $(x, y, z)$ by $\left(\frac{x}{3}, \frac{x}{3}, \frac{x}{3}\right)$ in $(2.1)$, we obtain

$$
\left|\frac{1}{3} f\left(\frac{x}{3}\right)-f(x)\right| \leq \phi\left(\frac{x}{3}, \frac{x}{3}, \frac{x}{3}\right) .
$$

Again replacing $x$ by $\frac{x}{3}$ in (2.2) and dividing by 3 we get

$$
\left|\frac{1}{3^{2}} f\left(\frac{x}{3^{2}}\right)-f(x)\right| \leq \phi\left(\frac{x}{3}, \frac{x}{3}, \frac{x}{3}\right)+\frac{1}{3} \phi\left(\frac{x}{3^{2}}, \frac{x}{3^{2}}, \frac{x}{3^{2}}\right) .
$$

Again replacing $x$ by $\frac{x}{3}$ in the above inequality and dividing by 3 we get

$$
\left|\frac{1}{3^{3}} f\left(\frac{x}{3^{3}}\right)-f(x)\right| \leq \sum_{i=0}^{2} \frac{1}{3^{i}} \phi\left(\frac{x}{3^{i+1}}, \frac{x}{3^{i+1}}, \frac{x}{3^{i+1}}\right) .
$$

Proceeding further and using induction on a positive integer $n$, we get

$$
\begin{aligned}
\left|\frac{1}{3^{n}} f\left(\frac{x}{3^{n}}\right)-f(x)\right| & \leq \sum_{i=0}^{n-1} \frac{1}{3^{i}} \phi\left(\frac{x}{3^{i+1}}, \frac{x}{3^{i+1}}, \frac{x}{3^{i+1}}\right) \\
& \leq \sum_{i=0}^{\infty} \frac{1}{3^{i}} \phi\left(\frac{x}{3^{i+1}}, \frac{x}{3^{i+1}}, \frac{x}{3^{i+1}}\right)
\end{aligned}
$$

for all $x \in X$. In order to prove the convergence of the sequence $\left\{\frac{1}{3^{n}} f\left(\frac{x}{3^{n}}\right)\right\}$, replace $x$ by $\frac{x}{3^{p}}$ in $(2.3)$ and divide by $3^{p}$, we find that for $n>p>0$

$$
\begin{aligned}
\left|\frac{1}{3^{p}} f\left(\frac{x}{3^{p}}\right)-\frac{1}{3^{n+p}} f\left(\frac{x}{3^{n+p}}\right)\right| & =\frac{1}{3^{p}}\left|f\left(\frac{x}{3^{p}}\right)-\frac{1}{3^{n}} f\left(\frac{x}{3^{n+p}}\right)\right| \\
& \leq \sum_{i=0}^{\infty} \frac{1}{3^{p+i}} \phi\left(\frac{x}{3^{p+i+1}}, \frac{x}{3^{p+i+1}}, \frac{x}{3^{p+i+1}}\right) \\
& \leq \sum_{i=p}^{\infty} \frac{1}{3^{i}} \phi\left(\frac{x}{3^{i+1}}, \frac{x}{3^{i+1}}, \frac{x}{3^{i+1}}\right) .
\end{aligned}
$$

Allow $p \rightarrow \infty$, the right-hand side of the above inequality tends to 0 . Thus the sequence $\left\{\frac{1}{3^{n}} f\left(\frac{x}{3^{n}}\right)\right\}$ is a Cauchy sequence. Thus we may 
define a mapping $A: X \rightarrow Y$ by

$$
A(x):=\lim _{n \rightarrow \infty} \frac{1}{3^{n}} f\left(\frac{x}{3^{n}}\right)
$$

for all $x \in X$. By (2.3) with $n \rightarrow \infty$, we have

$$
|f(x)-A(x)| \leq \Phi(x, x, x)
$$

for all $x \in X$. Replacing $(x, y, z)$ by $\left(\frac{x}{3^{n}}, \frac{y}{3^{n}}, \frac{z}{3^{n}}\right)$ in (2.1) and dividing by $3^{n}$ we obtain

$$
\begin{aligned}
& \frac{1}{3^{n}} \mid f\left(\frac{1}{3^{n}}\left(\frac{x+y+z}{3}\right)\right)-f\left(\frac{1}{3^{n}}(x+y+z)\right) \\
& \quad-\frac{2 f\left(\frac{u}{3^{n}}\right) f\left(\frac{v}{3^{n}}\right) f\left(\frac{w}{3^{n}}\right)}{f\left(\frac{u}{3^{n}}\right) f\left(\frac{v}{3^{n}}\right)+f\left(\frac{v}{3^{n}}\right) f\left(\frac{w}{3^{n}}\right)+f\left(\frac{w}{3^{n}}\right) f\left(\frac{u}{3^{n}}\right)} \mid \\
& \leq \frac{1}{3^{n}} \phi\left(\frac{x}{3^{n}}, \frac{y}{3^{n}}, \frac{z}{3^{n}}\right)
\end{aligned}
$$

for all $(x, y, z) \in X^{3}$, where $u=\frac{x+y}{2}, v=\frac{y+z}{2}$ and $w=\frac{z+x}{2}$. Letting $n \rightarrow \infty$, we have

$$
A\left(\frac{x+y+z}{3}\right)-A(x+y+z)=\frac{2 A(u) A(v) A(w)}{A(u) A(v)+A(v) A(w)+A(w) A(u)}
$$

for all $(x, y, z) \in X^{3}$, where $u=\frac{x+y}{2}, v=\frac{y+z}{2}$ and $w=\frac{z+x}{2}$. Now let $B: X \rightarrow Y$ be another function which satisfies the equation (1.1) and $|f(x)-B(x)| \leq \Phi(x, x, x)$ for all $x \in X$. Since $3^{n} B(x)=B\left(\frac{x}{3^{n}}\right)$ and $3^{n} A(x)=A\left(\frac{x}{3^{n}}\right)$, we have

$$
\begin{aligned}
|B(x)-A(x)| & =\frac{1}{3^{n}}\left|B\left(\frac{x}{3^{n}}\right)-A\left(\frac{x}{3^{n}}\right)\right| \\
& \leq \frac{1}{3^{n}}\left(\left|B\left(\frac{x}{3^{n}}\right)-f\left(\frac{x}{3^{n}}\right)\right|+\left|f\left(\frac{x}{3^{n}}\right)-A\left(\frac{x}{3^{n}}\right)\right|\right) \\
& \leq 2 \sum_{i=0}^{\infty} \frac{1}{3^{n+i}} \phi\left(\frac{x}{3^{n+i+1}}, \frac{x}{3^{n+i+1}}, \frac{x}{3^{n+i+1}}\right)
\end{aligned}
$$

for all $x \in X$. Allowing $n \rightarrow \infty$ in the above inequality, we find that $\mathrm{A}$ is unique. This completes the proof of the theorem.

Corollary 2. Let $X$ and $Y$ be spaces of non-zero real numbers. Assume that $f: X \rightarrow Y$ satisfies the functional inequality

$$
\left|D_{f}(x, y, z)\right| \leq \delta
$$


for all $x, y, z \in X$. Then there exists a unique multiplicative inverse mapping $A: X \rightarrow Y$ which satisfies (1.1) and

$$
|f(x)-A(x)| \leq 3 \delta
$$

for all $x \in X$.

Proof. Let $\phi(x, y, z)=\delta$ for all $x, y, z \in X$. Then by Theorem 1 ,

$$
\Phi(x)=\sum_{i=0}^{\infty} \frac{\delta}{3^{i}}=3
$$

for all $x \in X$. This completes the proof of the corollary.

Theorem 3. Let $X$ and $Y$ be spaces of non-zero real numbers. Assume that $f: X \rightarrow Y$ satisfies the functional inequality

$$
\left|D_{f}(x, y, z)\right| \leq \psi(x, y, z)
$$

for all $x, y, z \in X$, where $\psi: X^{3} \rightarrow Y$ is a function such that for all $x, y, z \in X$

$$
\Psi(x, y, z):=\sum_{i=0}^{\infty} 3^{i+1} \psi\left(3^{i} x, 3^{i} y, 3^{i} z\right)<\infty .
$$

Then there exists a unique multiplicative inverse mapping $A: X \rightarrow Y$ which satisfies (1.1) and

$$
|f(x)-A(x)| \leq \Psi(x, x, x)
$$

for all $x \in X$.

Proof. Replacing $(x, y, z)$ by $(x, x, x)$ in $(2.4)$ and multiplying by 3 , we obtain

$$
|f(x)-3 f(3 x)| \leq 3 \psi(x, x, x) .
$$

Again replacing $x$ by $3 x$ in (2.5) and multiplying by 3 we get

$$
\left|f(x)-3^{2} f\left(3^{2} x\right)\right| \leq 3 \psi(x, x, x)+3^{2} \phi(3 x, 3 x, 3 x) .
$$

Again replacing $x$ by $3 x$ in the above inequality and multiplying by 3 we get

$$
\left|f(x)-3^{3} f\left(3^{3} x\right)\right| \leq \sum_{i=0}^{2} 3^{i+1} \psi\left(3^{i} x, 3^{i} x, 3^{i}\right) .
$$


Proceeding further and using induction on a positive integer $n$, we get

$$
\begin{aligned}
\left|f(x)-3^{n} f\left(3^{n} x\right)\right| & \leq \sum_{i=0}^{n-1} 3^{i+1} \psi\left(3^{i} x, 3^{i} x, 3^{i} x\right) \\
& \leq \sum_{i=0}^{\infty} 3^{i+1} \psi\left(3^{i} x, 3^{i} x, 3^{i} x\right)
\end{aligned}
$$

for all $x \in X$. In order to prove the convergence of the sequence $\left\{3^{n} f\left(3^{n} x\right)\right\}$, replace $x$ by $3^{p} x$ in $(2.6)$ and divide by $3^{p}$, we find that for $n>p>0$

$$
\begin{aligned}
\left|3^{p} f\left(3^{p} x\right)-3^{n+p} f\left(3^{n+p} x\right)\right| & =3^{p}\left|f\left(3^{p} x\right)-3^{n} f\left(3^{n+p} x\right)\right| \\
& \leq \sum_{i=0}^{\infty} 3^{p+i+1} \phi\left(3^{p+i} x, 3^{p+i} x, 3^{p+i} x\right) \\
& \leq \sum_{i=p}^{\infty} 3^{i+1} \phi\left(3^{i} x, 3^{i} x, 3^{i} x\right) .
\end{aligned}
$$

Allow $p \rightarrow \infty$, the right-hand side of the above inequality tends to 0 . Thus the sequence $\left\{3^{n} f\left(3^{n} x\right)\right\}$ is a Cauchy sequence. Thus we may define a mapping $A: X \rightarrow Y$ by

$$
A(x):=\lim _{n \rightarrow \infty} 3^{n} f\left(3^{n} x\right)
$$

for all $x \in X$. By (2.6) with $n \rightarrow \infty$, we have

$$
|f(x)-A(x)| \leq \Psi(x, x, x)
$$

for all $x \in X$. Replacing $(x, y, z)$ by $\left(3^{n} x, 3^{n} y, 3^{n} z\right)$ in (2.4) and multiplying by $3^{n}$ we obtain

$$
\begin{aligned}
& 3^{n} \mid f\left(3^{n}\left(\frac{x+y+z}{3}\right)\right)-f\left(3^{n}(x+y+z)\right) \\
& \quad-\frac{2 f\left(3^{n} x\right) f\left(3^{n} y\right) f\left(3^{n} z\right)}{f\left(3^{n} x\right) f\left(3^{n} y\right)+f\left(3^{n} y\right) f\left(3^{n} z\right)+f\left(3^{n} z\right) f\left(3^{n} x\right)} \mid \\
& \leq \frac{1}{3} 3^{n+1} \psi\left(3^{n} x, 3^{n} y, 3^{n} z\right)
\end{aligned}
$$

for all $(x, y, z) \in X^{3}$. Since $\Psi(x, y, z)$ converses, letting $n \rightarrow \infty$, we have

$$
A\left(\frac{x+y+z}{3}\right)-A(x+y+z)=\frac{2 A(x) A(y) A(z)}{A(x) A(y)+A(y) A(z)+A(z) A(x)}
$$


for all $(x, y, z) \in X^{3}$. Now let $B: X \rightarrow Y$ be another function which satisfies the equation (1.1) and $|f(x)-B(x)| \leq \Psi(x, x, x)$ for all $x \in X$. Since $B\left(3^{n} x\right)=\frac{1}{3^{n}} B(x)$ and $A\left(3^{n} x\right)=\frac{1}{3^{n}} A(x)$ we have

$$
\begin{aligned}
|B(x)-A(x)| & =3^{n}\left|B\left(3^{n} x\right)-A\left(3^{n} x\right)\right| \\
& \leq 3^{n}\left(\left|B\left(3^{n} x\right)-f\left(3^{n} x\right)\right|+\left|f\left(3^{n} x\right)-A\left(3^{n} x\right)\right|\right) \\
& \leq 2 \sum_{i=0}^{\infty} 3^{n+i+1} \psi\left(3^{n+i} x, 3^{n+i} x, 3^{n+i} x\right)
\end{aligned}
$$

for all $x \in X$. Allowing $n \rightarrow \infty$ in the above inequality, we find that $\mathrm{A}$ is unique. This completes the proof of the theorem.

Theorem 4. Let $X$ and $Y$ be spaces of non-zero real numbers. Assume that $f: X \rightarrow Y$ satisfies the functional inequality

$$
\left|E_{f}(x, y, z)\right| \leq \phi(x, y, z)
$$

for all $x, y, z \in X$, where $\phi: X^{3} \rightarrow Y$ is a function such that for all $x, y, z \in X$

$$
\Phi(x, y, z):=\sum_{i=0}^{\infty} \frac{1}{3^{i}} \phi\left(\frac{x}{3^{i+1}}, \frac{y}{3^{i+1}}, \frac{z}{3^{i+1}}\right)<\infty .
$$

Then there exists a unique multiplicative inverse mapping $A: X \rightarrow Y$ which satisfies (1.2) and

$$
|f(x)-A(x)| \leq \Phi(x, x, x)
$$

for all $x \in X$.

Proof. Replacing $(x, y, z)$ by $\left(\frac{x}{3}, \frac{x}{3}, \frac{x}{3}\right)$ in (2.7), we obtain

$$
\left|\frac{1}{3} f\left(\frac{x}{3}\right)-f(x)\right| \leq \phi\left(\frac{x}{3}, \frac{x}{3}, \frac{x}{3}\right) \text {. }
$$

By the same method of proof in Theorem 1, we complete the proof.

Corollary 5. Let $X$ and $Y$ be spaces of non-zero real numbers. Assume that $f: X \rightarrow Y$ satisfies the functional inequality

$$
\left|E_{f}(x, y, z)\right| \leq \delta
$$

for all $x, y, z \in X$. Then there exists a unique multiplicative inverse mapping $A: X \rightarrow Y$ which satisfies (1.2) and the inequality

$$
|f(x)-A(x)| \leq 3 \delta
$$


for all $x \in X$.

Theorem 6. Let $X$ and $Y$ be spaces of non-zero real numbers. Assume that $f: X \rightarrow Y$ satisfies the functional inequality

$$
\left|E_{f}(x, y, z)\right| \leq \psi(x, y, z)
$$

for all $x, y, z \in X$, where $\psi: X^{3} \rightarrow Y$ is a function such that for all $x, y, z \in X$

$$
\Psi(x, y, z):=\sum_{i=0}^{\infty} 3^{i+1} \psi\left(3^{i} x, 3^{i} y, 3^{i} z\right)<\infty .
$$

Then there exists a unique multipicative inverse mapping $A: X \rightarrow Y$ which satisfies (1.2) and the inequality

$$
|f(x)-A(x)| \leq \Psi(x, x, x)
$$

for all $x \in X$.

Proof. Replacing $(x, y, z)$ by $(x, x, x)$ in (2.8) and multiplying by 3 , we obtain

$$
|f(x)-3 f(3 x)| \leq 3 \psi(x, x, x) .
$$

By the same method of proof in Theorem 3, we complete the proof.

\section{References}

[1] J. Baker, The stability of the cosine equations, Proc. Amer. Math. Soc. 80 (1980), 411-416.

[2] J. Baker, J. Lawrence and F. Zorzitto, The stability of the equation $f(x+y)=$ $f(x)+f(y)$, Proc. Amer. Math. Soc. 74 (1979), 242-246.

[3] G. L. Forti, Hyers-Ulam stability of functional equations in several variables, Aequationes Math. 50 (1995), 146-190.

[4] P. Găvruta, A generalization of the Hyers-Ulam-Rassias stability of approximately additive mappings, J. Math. Anal. Appl., 184 (1994), 431-436.

[5] D.H. Hyers, On the stability of the linear functional equation, Proc. Nat. Acad. Sci. U. S. A. 27 (1941), 222-224.

[6] D.H. Hyers, G. Isac, and Th.M. Rassias, Stability of functional equations in several variables, Birkhäuser-Basel-Berlin(1998).

[7] K.W. Jun, G.H. Kim and Y.W. Lee, Stability of generalized gamma and beta functional equations, Aequation Math. 60(2000), 15-24.

[8] S.-M. Jung, On the general Hyers-Ulam stability of gamma functional equation, Bull. Korean Math. Soc. 34 No 3 (1997), 437-446.

[9] S.-M. Jung, On the stability of the gamma functional equation, Results Math. 33 (1998), 306-309. 
[10] G.H. Kim, and Y.W. Lee, The stability of the beta functional equation, BabesBolyai Mathematica, XLV (1) (2000), 89-96.

[11] Y.W. Lee, On the stability of a quadratic Jensen type functional equation, J. Math. Anal. Appl. 270 (2002) 590-601.

[12] Y.W. Lee and G.H. Kim, Stability of the reciprocal difference and adjoint functional equations in m-variables, J. of the Chungcheong Math. Soc. 23 (2010), 731-739.

[13] Th.M. Rassias, On the stability of the linear mapping in Banach spaces, Proc. Amer. Math. Soc. 72 (1978), 297-300.

[14] K. Ravi, J. M. Rassias and B. V. Senthil Kumar, lam stability of reciprocal difference and adjoint functional equqtions, to appear.

[15] S.M. Ulam, Problems in Modern Mathematics, Proc. Chap. VI. Wiley. NewYork, 1964.

\section{Eun Hwi Lee}

Department of Mathematics, Jeonju University, Jeonju 560-759, Korea.

E-mail: ehl@jj.ac.kr 